\title{
NOTES OF A CASE OF IMPERFORATE RECTUM; INGUINAL COLOTOMY; DEATH.
}

\author{
BY WILLIAM BERRY, F.R.C.S.; \\ Hon. Surgeon, Royal Albert Edward Infirmary, Wigan. \\ [Read in the Pathological Section, May 3, 1889].
}

I was called to see this case by Dr. Foreman, who had seen the child three days after its birth, and had tried several times to get a passage by passing a large bougie into the rectum. When I saw the child it was six days old, was fairly well nourished, and of average size. Everything it took in the shape of diluted milk, and occasionally the breast milk, remained on its stomach.

I examined and found the anus patent, but on introducing my little finger I could not pass it more than two inches, the occlusion appearing to be at the commencement of the rectum. I then tried to pass a No. 10 gum elastic catheter, but without avail. We had apparently a case in a female of the fourth variety of rectal malformation (Papendorf's classification), as given by Dr. Ball in his excellent work on the "Rectum and Anus," p. 31, where he gives a good illustration. He states that " these cases are especially interesting, as they unquestionably indicate that the malformation is due to the fact that the procto-dæum has failed to meet the mesenteron, and that they cannot possibly be due to an obliteration of the rectum after complete development."-Opus cit., p. 32. Operation was at this time suggested but refused.

On the eighteenth day, the child now getting restless, uneasy, and suffering from abdominal distension, but without vomiting, the parents consented to an operation.

On Feb. 6th Dr. Foreman administered chloroform, and assisted me to perform inguinal colotomy in the left groin. We thought this would be more satisfactory than trying to find the commence- 
ment of the rectum, and necessitating colotomy as a last resort. There was not much difficulty in finding the colon, which was sutured to the incision and opened. A large amount of flatus and some fæcal matter, but little meconium, escaped, An absorbent pad was placed over the wound, and the child wrapped in flannel. Next day the opening looked well. The child had vomited, probably from effects of chloroform.

Feb. 8th.-The wound looked all right. Some fæces had passed, and the child seemed fairly well, the vomiting having ceased.

Feb. 9th.-The wound was red and dusky, the surrounding tissue red and gangrenous-looking. The child died in the afternoon, apparently from exhaustion.

No post-mortem was allowed.

This case is especially interesting, from the fact of the child living for nineteen days without any passage through its bowels. Great care had been exercised not to overload the stomach. There was very little wasting up to the time of the operation. If the operation had been done early, it is possible we should have had a favourable result. 